\title{
Mean free path of a suddenly created fast electron moving in a degenerate electron gas
}

\author{
I. Nagy ${ }^{1,2}$ and P. M. Echenique ${ }^{2,3}$ \\ ${ }^{1}$ Department of Theoretical Physics, Institute of Physics, Technical University of Budapest, H-1521 Budapest, Hungary \\ ${ }^{2}$ Donostia International Physics Center, P. Manuel de Lardizabal 4, E-20018 San Sebastián, Spain \\ ${ }^{3}$ Departamento de Física de Materiales, Facultad de Químicas, Universidad del Pais Vasco, Apto. 1072, E-20018 San Sebastián, Spain
}

(Received 26 September 2011; revised manuscript received 30 January 2012; published 29 March 2012)

\begin{abstract}
A lower bound on the mean free path $(l)$ of a swift electron moving in a degenerate electron gas is calculated by implementing a standard theoretical framework for the collision rate, $1 / \tau$, with a scattering amplitude characterized by the matrix element of a hole-screened interaction potential taken between plane-wave states. The instantaneous hole around a system's electron is considered at the Hartree-Fock level for the ground-state wave function of the degenerate electron gas. The real transitions in the many-body system are considered by following Galitskii's treatment on an almost perfect Fermi gas of neutral atomic constituents. The analytical results show minima both in $\tau$ and $l$, and they appear at $\left(E / E_{F}\right) \simeq 4.6$ and $\left(E / E_{F}\right) \simeq 2.5$, respectively, where $E$ is the kinetic energy of the fast electron and $E_{F}$ is the Fermi energy of the host. Comparison with mean free path data obtained recently for $\mathrm{Cu}$ is made and a reasonable agreement is found.
\end{abstract}

DOI: 10.1103/PhysRevB.85.115131

\section{INTRODUCTION}

The many-body problem is a topic in its own right, with its own characteristic methods. Particularly, the model system of identical fermion particles, not localized in space and subject to Pauli's exclusion principle, represents a genuinely important problem and the dynamical probing of correlated motions of constituents of such a many-body system is currently an active subfield in physics. In order to put the present study on inelastic scattering into a proper perspective, we follow below the wellknown ${ }^{1}$ historical path of developments whose result is named in the literature as Landau's Fermi liquid theory.

By considering the liquid state of the rare fermionic isotope of neutral helium atoms, Landau concluded that the low-lying excited states of such a system can be described by quasiparticles. Implications for an electron gas were immediately (see Sec. II) recognized; the essential difference being the presence of charged free particles instead of neutral atoms. It is this difference that requires different modeling of an effective interaction in the mean free path calculation of an electron in the electron gas, and is the subject of our study.

In his pioneering work on applying a field-theoretic ${ }^{2}$ Green's function method to a nonrelativistic many-body system of neutral atomic fermions, Galitskii derived ${ }^{3}$ the following expression for the single-particle $\left[E=\left(v^{2} / 2 m\right) \geqslant E_{F}\right]$ scattering rate

$$
\frac{1}{\tau}=n_{0} \frac{3 v_{F}}{16 \pi}\left[V^{2} \frac{8}{15} \frac{1}{\sqrt{x}}\left(\Theta(2-x)(2-x)^{5 / 2}+\frac{5 x-7}{2}\right)\right],
$$

where $x \equiv E / E_{F}$ in terms of the Fermi energy $E_{F}=k_{F}^{2} / 2$, and $n_{0}=k_{F}^{3} /\left(3 \pi^{2}\right)$ is the host particle density. $V \equiv 4 \pi a_{\mathrm{sc}}$, where $a_{\mathrm{sc}}$ is a scattering length characterizing the two-body collision. We employ Hartree atomic units, $e^{2}=\hbar=m_{e}=1$, throughout this work. For the low-energy (i.e., $x \rightarrow 1^{+}$limit) one gets a quadratic

$$
\frac{1}{\tau}=\left[n_{0} \frac{3}{4}\left(\frac{E-E_{F}}{E_{F}}\right)^{2}\right] v_{F} \frac{V^{2}}{4 \pi}
$$

PACS number(s): 71.10.Ca, 71.10.Li, 79.20.Uv, 79.60.-i

dependence due to mainly the Pauli's exclusion principle. The number of active particles is transparent in such a form. For a fast particle moving with velocity $v \gg v_{F}$, we get

$$
\frac{1}{\tau}=n_{0}\left(4 \pi a_{\mathrm{sc}}^{2}\right) v \text {. }
$$

This asymptotic form shows a classically expected ${ }^{4}$ averaged rate for an individual intruder particle that collides with independent particles of the fermion system at high relative velocity $v$ in a cumulative manner with a constant area, $4 \pi a_{\mathrm{sc}}^{2}$, for binary interactions. It is easy to show that a mean free path (l) at fixed $n_{0}$, defined by

$$
l(v)=v \tau(v),
$$

tends to a constant value from above in a monotonously decreasing way as a function of increasing $v \geqslant v_{F}$. This is due to the assumption ( $\left.\propto a_{\text {sc }}\right)$ on the scattering amplitude.

The low-energy limit [i.e., Eq. (2)] shows a quadratically vanishing scattering rate. A quadratic form for the rate in terms of the excess $\left[\Delta E \equiv\left(E-E_{F}\right)\right]$ energy is, of course, much smaller than the energy $E \geqslant E_{F}$ of the added particle. Such a quadratic dependence is in harmony with one of the well-known ${ }^{5}$ limiting forms

$$
\frac{\alpha}{E-E_{F}} \leqslant \tau(E) \leqslant \frac{\beta}{\left(E-E_{F}\right)^{2}},
$$

between which a quasiparticle concept is justified. The problem of lifetime, mediated by quasiparticle-quasiparticle interaction, was analyzed in detail ${ }^{6}$ in a perturbative manner by Luttinger using skeleton diagrams to find a proper self-energy of a particlelike excitation. It was pointed out that with wellbehaved local interaction one gets a quadratic dependence for $1 / \tau$ in the excess energy (i.e., a proportionality to $\left(E-E_{F}\right)^{2}$ even in infinite order of perturbation theory) but no general (i.e., necessary and sufficient) conditions on the interaction form was found for which this characteristic dependence is valid.

The key point in the mentioned nontrivial analysis, with a presumed well-behaved interaction, is that the conservation laws could determine alone a quadratic dependence. With 
such an interaction one can (see Sec. II) consider separately the double integration in transferred momenta and energies at low enough excess energies, $\left(E-E_{F}\right)$. However, in a second-order description for the scattering rate the internal frequencies should ${ }^{3,7}$ correspond to the energies of two real colliding particles. Thus the conservation laws, together with the more restrictive Pauli's exclusion principle for allowed real transitions, determine uniquely the connection between transferred momentum $q$ and energy $\omega \geqslant 0$ in scattering.

After these fine details in a many-body problem, we turn to our practical approximation on which the results and our statements are based. These are given in the next section. The last section, Sec. III, contains a brief summary and our remarks.

\section{RESULTS AND DISCUSSION}

Following closely our previous ${ }^{8}$ re-derivation of Galitskii's result in Eq. (1), we use a textbook ${ }^{9}$ expression for the net rate of creating electron-hole pairs, and write

$$
\frac{1}{\tau}=n_{0} \frac{3 v_{F}}{16 \pi} \frac{1}{\sqrt{x}} \int_{0}^{x-1} d y \int_{u_{-}}^{u_{+}} d u[V(q)]^{2} F^{0}(u, y),
$$

in terms of dimensionless variables defined by $y=\omega / E_{F}$, $u=q / k_{F}$, and $x=E / E_{F}$. The above form shows that we will use an instantaneous interaction $V\left(q=u k_{F}\right)$. The systemdependent kernel function $F^{0}(u, y)$ for positive $\omega$ is given ${ }^{9}$ by the following form

$$
F^{0}(u, y)=\left[\Theta\left(1-P_{1}\right)\left(1-P_{1}^{2}\right)-\Theta\left(1-P_{2}\right)\left(1-P_{2}^{2}\right)\right]
$$

where $P_{1}=(1 / 2 u)\left|y-u^{2}\right|$ and $P_{2}=(1 / 2 u)\left(y+u^{2}\right)$. As we explained in Sec. I, the limits $u_{-}$and $u_{+}$are determined by the constraints encoded in $F^{0}(u, y)$ at a fixed $y$. For instance, for $x \in[1,2]$ the second constraint prescribes a $(1-\sqrt{1-y}) \leqslant$ $u \leqslant(1+\sqrt{1-y})$ range for allowed momentum transfer. It is easy to derive the limits, valid now for all $x \geqslant 1$, due to the other constraint as well. One gets $(\sqrt{1+y}-1) \leqslant u \leqslant$ $(\sqrt{1+y}+1)$ for this case.

A finite lifetime is due to real transitions in the system, which are characterized via an ideal $\left[\propto F^{0}(q, \omega)\right]$ dynamical structure function, where the variables $\omega$ and $q$ are energy and momentum transfers the source of which are the energy and momentum of an added energetic electron. Now, if one considers $^{10-12}$ the many-body system as a charge-polarizable medium, one may use a dielectric function $\varepsilon(k, \Omega)$ in which the wave vector and frequency (i.e., the Fourier variables, $k$ and $\Omega$, in a space-time-dependent description of density fluctuations) are fixed ${ }^{13}$ to the above kinematical variables. In such a modeling of matrix elements one has $|V(q, \omega)|=$ $\left(4 \pi / q^{2}\right) /|\varepsilon(q, \omega)|$. Thus, at small excess energy $(\omega \rightarrow 0)$, one gets the conventional mean-field screening of a fixed classical point charge.

Unfortunately, the proper incorporation of statisticsdependent correlation effects into a quasiclassical chargepolarization picture on effective electron-electron interaction is a highly debated issue. ${ }^{14}$ In simple terms, one can not, without an unphysical overcounting, superpose two normalized holes around a system's electron, which are due to, separately, electrostatics and quantum mechanical exchange. Moreover, there is an accumulating experimental evidence ${ }^{15-19}$ that a dielectric modeling results in inelastic mean free paths $l(E)$, which are far from both the experimental minimum values and their energetic positions. It is this theoretical and experimental background on which we return to a basic quantum mechanical model, without electrostatic polarization, in order to discuss the inelastic mean free path of an energetic electron in an electron gas.

Since the aim of this study is to derive a lower bound on the mean free path $(l)$ of an added free electron with $v \geqslant v_{F}$, we will use a model that still respects a basic aspect of the degenerate electron gas in a charge-compensating background. The ground state is a Slater determinant of single-particle plane-wave states. Thus, due to Pauli's exclusion principle, there is a unit-norm exchange hole ${ }^{9,20}$ around each moving system electron. In other words, the total missing charge from such a hole is one electron charge. This statistical distribution of system electrons around a given electron is a distribution of electrically charged particles, and therefore gives rise to an electric potential. Since the quantum hole efficiently quenches ${ }^{20,21}$ the quasiclassical polarization action of other electrons around a given electron, this action is neglected (cf., the discussion of Fig. 2) in the present work.

Thus, we model the pair interaction based on the above first-order knowledge. Since a scattering rate $(1 / \tau)$ is a second-order quantity, as Eq. (4) shows, our construction is consistent. Such a physically motivated modeling was employed successfully ${ }^{22}$ earlier to describe the change in the pair-correlation function. We start, therefore, by taking

$$
V(q)=\frac{4 \pi}{q^{2}} \frac{3}{4} \frac{q}{k_{F}}\left(1-\frac{1}{12} \frac{q^{2}}{k_{F}^{2}}\right),
$$

for $q \leqslant 2 k_{F}$ with $V(q)=4 \pi / q^{2}$ for $q>2 k_{F}$. Next, in order to derive a lower bound within this framework for $l(E)$ we take only the small- $q$ limit of the above expression. This is not a strong assumption since the scattering rate $1 / \tau$ is dominantly sensitive to the $q \rightarrow 0$ limit, the forward direction in scattering. With $V\left(u, k_{F}\right)=\left[3 \pi / k_{F}^{2}\right] / u$ in Eq. (4), the somewhat lengthy integrations are still analytic. We obtain for this case

$$
\frac{1}{\tau}=n_{0} \frac{3 v_{F}}{16 \pi}\left[\left(\frac{3 \pi}{k_{F}^{2}}\right)^{2} \frac{4}{3} \frac{g_{1}(x)-g_{2}(x)}{\sqrt{x}}\right] .
$$

The functions, $g_{1}(x)$ and $g_{2}(x)$, are given by the following expressions

$$
\begin{aligned}
g_{1}(x)= & \ln (x-1)+\left(\frac{8}{3}-2 \ln 2\right), \\
g_{2}(x)= & \Theta(2-x) \\
& \times\left[\frac{2}{3}(2-x)^{3 / 2}+2(2-x)^{1 / 2}+\ln \left|\frac{\sqrt{2-x}-1}{\sqrt{2-x}+1}\right|\right] .
\end{aligned}
$$

As it is well known, a pure $1 / q^{2}$ dependence for all $q$ values in $V(q)$ would result in a strongly divergent expression for $1 / \tau$, and thus in a vanishing mean free path. Our method shows a quantum-mechanics-governed way to remove this unphysical divergency.

Using the above result in Eq. (7) for the scattering rate, the mean free path becomes

$$
l(v)=v \tau(v)=\frac{4 \pi}{3} v_{F} \frac{x}{g_{1}(x)-g_{2}(x)} .
$$




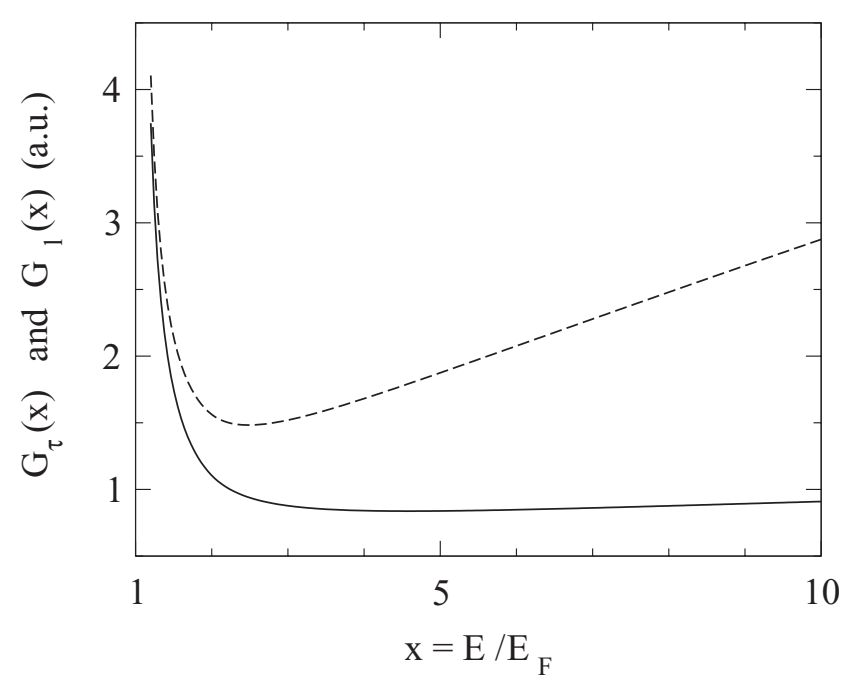

FIG. 1. The $G_{\tau}(x)$ (solid curve) and the $G_{l}(x)$ (dashed curve) functions in atomic units, for the range $x \in[1.1,10]$ of the dimensionless variable $x=E / E_{F}$. See the text for further details.

There are minimum ( $m$ ) values in $\tau(x)$ and $l(x)$ at a fixed parameter $v_{F}$. These appear at the solutions of the algebraic equations $(x-1)[(8 / 3)-2 \ln 2+\ln (x-1)]=2 x$ and $(x-1)$ $[(8 / 3)-2 \ln 2+\ln (x-1)]=x$, respectively. By a simple calculation, we get $x_{m}^{\tau} \simeq 4.6$ and $x_{m}^{l} \simeq 2.5$, respectively. Thus, $\tau\left(x_{m}^{\tau}=4.6\right) \simeq 3.3$ and $l\left(x_{m}^{l}=2.5\right) \simeq 6.2 v_{F}$, both in atomic units. For a typical metal $\left(v_{F} \simeq 1\right)$ the corresponding values are about $l \simeq 3 \AA$ and $\tau \simeq 8 \times 10^{-17}$ seconds (i.e., 80 attoseconds). For illustration, in Fig. 1 we exhibit, by considering the simple structure of Eq. (8), the following functions

$$
G_{\tau}(x)=\frac{\sqrt{x}}{g_{1}(x)-g_{2}(x)}, \quad G_{l}(x)=\frac{x}{g_{1}(x)-g_{2}(x)},
$$

as a function of the convenient, scaled variable $x=\left(E / E_{F}\right)$ for $x \in[1.1,10]$. Notice at this point that incorporation of band $(b)$ effects ${ }^{17,18}$ on our free-electron velocity could result in, presumably, a monotonous $v_{b}(E) \leqslant \sqrt{2 E}$ function. Thus, with such a $v_{b}(E)$ the minimum in our $l(E)$ would appear between the above $x_{m}^{i}$ values.

Contrary to the solution in Eq. (1), obtained by Galitskii with a momentum-independent matrix element, our $l(x)=$ $(4 \pi / 3) v_{F} G_{l}(x)$ function is not monotonic in $x=E / E_{F}$. This is due to, partly, the nonmonotonic $\tau(E)=(4 \pi / 3) G_{\tau}(x)$ function for the lifetime. The above compact result in Eq. (7) signals that even with a weakly $(\sim 1 / q)$ singular amplitude the whole problem is still an integrable one. This is an important observation of general interest in the light of Luttinger's statement on the allowed class of interactions. Furthermore, by a simple Taylor expansion of Eq. (7) we get

$$
\frac{1}{\tau}=\frac{3}{2 \pi} \frac{\left(E-E_{F}\right)}{E_{F}},
$$

for the case of $\left(E-E_{F}\right) \rightarrow 0$. Therefore, a $\hbar / \tau$ quantity is still much smaller than the kinetic energy of the particle with $E \simeq E_{F}$. The inverse of the above expression is an explicit lower-limit form to Pines's general ${ }^{5}$ bracketing (see Sec. I) for $\tau(E)$.

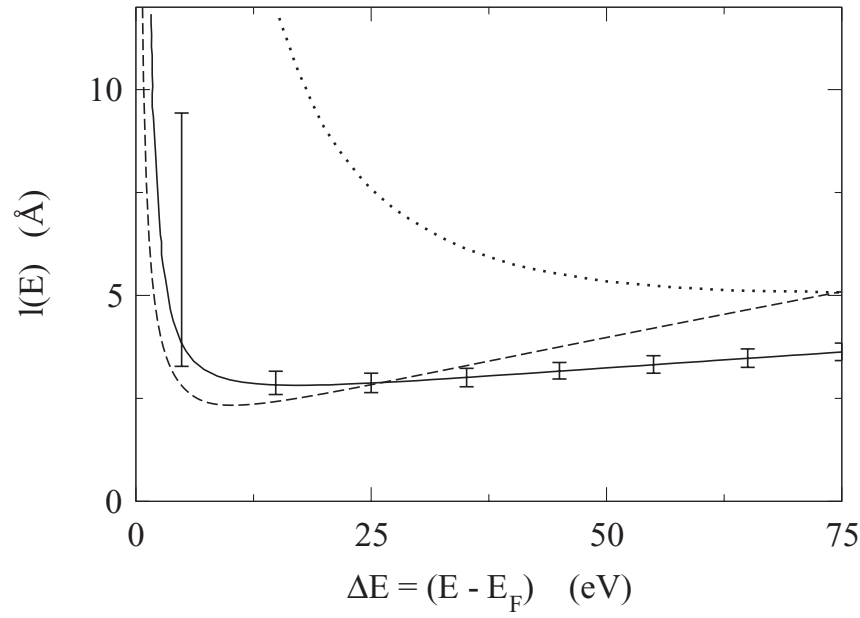

FIG. 2. The inelastic mean free path $l(E)$ as function of the photoelectron energy $\Delta E \equiv\left(E-E_{F}\right)$. The curve with error bars shows the experimental ${ }^{19}$ quantity. The dashed curve is based on the present theory. The dotted curve refers to a theoretical description that rests on an optical dielectric function for copper. See the text for further details.

Now we turn to the practical application of our theoretical results, by making a quantitative comparison with recent experimental data ${ }^{19}$ on inelastic mean free path in copper. We take $r_{s}=2.7$ for the Wigner-Seitz density parameter of the model system (i.e., we use the conduction band electron density). Thus one has $E_{F} \simeq 6.9 \mathrm{eV}$. In Fig. 2 our result is plotted by a dashed curve for the inelastic mean free path $l(E)$, while the other curve with error bars refers to the experiment. The dotted curve is based on a conventional ${ }^{23}$ theoretical modeling, which rests on an all-electron optical dielectric loss function.

It was emphasized in Ref. 19 that within such a conventional framework the position of the minimum in the mean free path is at too high photoelectron energies. Our present result (dashed curve) is in a reasonable agreement with data, considering the experimental error bar at the low-energy limit. In the most important range (i.e., around the experimental minimum) our curve follows the shape of the data. There is, however, a crossing between curves at around $E \simeq 33 \mathrm{eV}$. We speculate that this might be due to, at least partly, the growing importance of valence electrons. Furthermore, since the bosonic quantum $\left(\hbar \omega_{p}\right)$ should have some impact on the energy loss and mean free path, the proper inclusion of the collective polarization channel (neglected in this work) needs future considerations as well.

We finish our study by a qualitative analysis related to a recent hot topic. Streaked photoemission metrology 24 allows the observation of a relative time delay between the detection of photoelectrons from different initial electronic states. Theoretical attempts in this field are based ${ }^{25,26}$ on a time-dependent one-particle Schrödinger equation to explain the observed ${ }^{27}$ subfemtosecond time delay of energetic photoelectrons escaping from a metal, tungsten. Crucial in such studies, as an input parameter of many-body origin, is the inelastic mean free path to model the damping in a propagating wave. 
For instance, a small adjusted value of about $2.5 \AA$ was used $^{25}$ to reproduce the experimentally observed delay value for $W$. However, when a photoelectron releases from an atomic state, or from a solid environment, the combined fields of the targets and the streaking infrared (IR) pulse could influence $^{28,29}$ the elastic propagation in a highly nontrivial manner. Still, in our opinion, the above-discussed many-body inelasticity can be vital in quantitative interpretations of the delay data obtained for metals.

The analytical lower bound results on $\tau(E)$ and $l(E)$ could be useful, for instance, in computer-based simulation methods ${ }^{30}$ on the streaking phenomenon as well. The inverse of the total mean free path in a metal is taken usually as the sum of the elastic and inelastic inverses. The elastic component is, for a $50-100 \mathrm{eV}$ photoelectron energy range, in the $1-2 \AA$ interval according to a microscopic simulation work ${ }^{30}$ on tungsten. An only moderately higher magnitude for the inelastic component could have impact on the simulation of electron transport by using such a definition of the total mean free path.

\section{CONCLUSION}

Motivated by the importance of the inelastic mean free path $l(E)$ of suddenly generated energetic electrons in metals, a lower bound on $l(E)$ is derived in the present theoretical study. The study rests on the physical relevance ${ }^{20}$ of an exchange hole in the fermionic many-body system in order to characterize a scattering amplitude in momentum space. This input interaction was used in a second-order perturbation theory on many-body excitations. Our study extends the mathematical class of allowed pair interactions for which the lifetime and inelastic mean free path are still reasonable from physical $^{5}$ point of view.

We stress that both Eqs. (1) and (7) encode the effect of the fundamental exclusion principle of Pauli. But the details of encoding are different, since in our study on a charged fermionic system the principle is built into particle shielding as well, beyond the statistics-made restriction for allowed transitions. In other words, an interesting twofold role of quantum statistics is manifested in the present work for observable averages. As a final remark we note that further, consistent attempts are needed to incorporate a quasiclassical plasma screening (and, in particular, to understand the precise role of a plasmon-related collective inelastic channel) besides our quantum mechanical modeling of a dressed interaction.

\section{ACKNOWLEDGMENTS}

The authors thank A. K. Kazansky and E. E. Krasovskii for useful discussions. I.N. acknowledges the hospitality at the Donostia International Physics Center (DIPC) where the study was completed. This work was supported by the Spanish Ministry of Science and Innovation (MICINN) (Project No. FIS2010-19609-C02-02).
${ }^{1}$ W. Kohn, Rev. Mod. Phys. 71, S59 (1999).

${ }^{2}$ V. M. Galitskii and A. B. Migdal, Sov. Phys. JETP 34, 96 (1958).

${ }^{3}$ V. M. Galitskii, Sov. Phys. JETP 34, 279 (1958).

${ }^{4}$ L. P. Kadanoff and G. Baym, Quantum Statistical Mechanics (Perseus, Cambridge, 1962).

${ }^{5}$ D. Pines, in The Many-Body Problem (Benjamin, New York, 1961), pp. 31-34.

${ }^{6}$ J. M. Luttinger, Phys. Rev. 121, 942 (1961).

${ }^{7}$ E. M. Lifshitz and L. P. Pitaevskii, Statistical Physics, Vol. 2 (Pergamon, Oxford, 1980).

${ }^{8}$ I. Nagy, M. Alducin, and P. M. Echenique, Phys. Rev. B 65, 235102 (2002).

${ }^{9}$ G. D. Mahan, Many-Particle Physics (Plenum, New York, 1981).

${ }^{10}$ J. J. Quinn and R. A. Ferrell, Phys. Rev. 112, 812 (1958).

${ }^{11}$ R. H. Ritchie, Phys. Rev. 114, 644 (1959).

${ }^{12}$ S. Engelsberg, Phys. Rev. 123, 1130 (1961).

${ }^{13}$ D. Pines and P. Nozieres, in The Theory of Quantum Liquids (Benjamin, New York, 1966), pp. 243-245.

${ }^{14}$ G. D. Mahan, Comments Condens. Matter Phys. 16, 333 (1994).

${ }^{15}$ L. I. Johansson and B. E. Sernelius, Phys. Rev. B 50, 16817 (1994), and references therein.

${ }^{16}$ T.-W. Pi, I.-H. Hong, C.-P. Cheng, and G. K. Wertheim, J. Electron Spectroscopy Relat. Phenomena 107, 163 (2000).

${ }^{17}$ J. S. Dolado, V. M. Silkin, M. A. Cazalilla, A. Rubio, and P. M. Echenique, Phys. Rev. B 64, 195128 (2001).
${ }^{18}$ V. M. Silkin, E. V. Chulkov, and P. M. Echenique, Phys. Rev. B 68, 205106 (2003).

${ }^{19}$ J. D. Bourke and C. T. Chantler, Phys. Rev. Lett. 104, 206601 (2010).

${ }^{20}$ R. P. Feynman, in Statistical Mechanics (Perseus, Cambridge, 1972), pp. 245-250.

${ }^{21}$ J. M. Blatt, in Theory of Superconductivity (Academic, New York, 1964), pp. 120-122.

${ }^{22}$ M. Corona, P. Gori-Giorgi, and J. P. Perdew, Phys. Rev. B 69, 045108 (2004).

${ }^{23}$ Z.-J. Ding and R. Shimizu, Surf. Sci. 222, 313 (1989).

${ }^{24}$ F. Krausz and M. Ivanov, Rev. Mod. Phys. 81, 163 (2009).

${ }^{25}$ C.-H. Zhang and U. Thumm, Phys. Rev. Lett. 102, 123601 (2009); 103, 239902(E) (2009).

${ }^{26}$ A. K. Kazansky and P. M. Echenique, Phys. Rev. Lett. 102, 177401 (2009).

${ }^{27}$ A. L. Cavalieri, N. Müller, Th. Uphues, V. S. Yakovlev, A. Baltuska, B. Horvath, B. Schmidt, L. Blümel, R. Holzwarth, S. Hendel, M. Drescher, U. Kleineberg, P. M. Echenique, R. Kienberger, F. Krausz, and U. Heinzmann, Nature (London) 449, 1029 (2007).

${ }^{28}$ S. Nagele, R. Pazourek, J. Feist, K. Doblhoff-Dier, C. Lemell, K. Tőkési, and J. Burgdörfer, J. Phys. B 44, 081001 (2011).

${ }^{29}$ E. E. Krasovskii, Phys. Rev. B 84, 195106 (2011).

${ }^{30}$ C. Lemell, B. Solleder, K. Tőkési, and J. Burgdörfer, Phys. Rev. A 79, 062901 (2009). 